\title{
Treatment of diet-induced lipodystrophic C57BL/6J mice with long-acting PASylated leptin normalises insulin sensitivity and hepatic steatosis by promoting lipid utilisation
}

\author{
Florian Bolze ${ }^{1,2} \cdot$ Andrea Bast $^{1,2} \cdot$ Sabine Mocek ${ }^{1,2} \cdot$ Volker Morath $^{3} \cdot$ Detian Yuan $^{4}$. \\ Nadine Rink ${ }^{1,2}$ • Martin Schlapschy ${ }^{3}$ - Anika Zimmermann ${ }^{1,2} \cdot$ Mathias Heikenwalder $^{4}$. \\ Arne Skerra $^{3,5}$ - Martin Klingenspor ${ }^{1,2}$
}

Received: 10 March 2016 / Accepted: 11 May 2016/Published online: 7 June 2016

(C) Springer-Verlag Berlin Heidelberg 2016

\begin{abstract}
Aims/hypothesis Recombinant leptin offers a viable treatment for lipodystrophy (LD) syndromes. However, due to its short plasma half-life, leptin replacement therapy requires at least daily subcutaneous (s.c.) injections. Here, we optimised this treatment strategy in LD mice by using a novel leptin version with extended plasma half-life using PASylation technology. Methods A long-acting leptin version was prepared by genetic fusion with a 600 residue polypeptide made of Pro, Ala and Ser (PASylation), which enlarges the hydrodynamic volume and, thus, retards renal filtration, allowing less frequent injection. LD was induced in C57BL/6J mice by feeding a diet supplemented with conjugated linoleic acid (CLA). Chronic and acute effects of leptin treatment were assessed by evaluating plasma
\end{abstract}

Electronic supplementary material The online version of this article (doi:10.1007/s00125-016-4004-6) contains peer-reviewed but unedited supplementary material, which is available to authorised users.

\section{Arne Skerra \\ skerra@tum.de \\ Martin Klingenspor \\ mk@tum.de}

1 Lehrstuhl für Molekulare Ernährungsmedizin and Else Kröner-Fresenius Center, Technische Universität München, Gregor-Mendel-Str. 2, 85354 Freising (Weihenstephan), Germany

2 Research Center for Nutrition and Food Science (ZIEL), Technische Universität München, Freising (Weihenstephan), Germany

3 Munich Center for Integrated Protein Science (CIPS-M) and Lehrstuhl für Biologische Chemie, Technische Universität München, Emil-Erlenmeyer-Forum 5, 85354 Freising (Weihenstephan), Germany

4 Division of Chronic Inflammation and Cancer, German Cancer Research Center (DKFZ), Heidelberg, Germany

5 XL-protein GmbH, Freising, Germany insulin levels, insulin tolerance, histological liver sections, energy expenditure, energy intake and body composition.

Results In a cohort of female mice, 4 nmol PAS-leptin (applied via four s.c. injections every 3 days) successfully alleviated the CLA-induced LD phenotype, which was characterised by hyperinsulinaemia, insulin intolerance and hepatosteatosis. The same injection regimen had no measurable effect when unmodified recombinant leptin was administered at an equivalent dose. In a cohort of LD males, a single s.c. injection of PAS-leptin did not affect energy expenditure but inhibited food intake and promoted a shift in fuel selection towards preferential fat oxidation, which mechanistically substantiates the metabolic improvements.

Conclusions/interpretation The excellent pharmacological properties render PASylated leptin an agent of choice for refining both animal studies and therapeutic strategies in the context of LD syndromes and beyond.

Keywords Conjugated linoleic acid · Fuel selection · Leptin . Lipodystrophy $\cdot$ Metabolic rate $\cdot$ PASylation $\cdot$ Therapeutic protein
Abbreviations
ALT Alanine aminotransferase
AST Aspartate aminotransferase
CD Control diet
CLA Conjugated linoleic acid
HE Haematoxylin/eosin
LD Lipodystrophy
MR Metabolic rate
RER Respiratory exchange ratio 


\section{Introduction}

Lipodystrophies (LD) comprise a heterogeneous set of rare metabolic syndromes, either acquired or inherited in nature, which are characterised by a partial or generalised lack of adipose tissue [1]. In LD patients, the major functions of adipose tissue (i.e. storage of energy as triacylglycerols and release of adipokine hormones, such as leptin and adiponectin, to control energy and glucose homeostasis) are hampered [2, 3]. The limited storage capacity of adipose tissue leads to an ectopic fat deposition predominantly in the liver, pancreas and muscle. In addition, adipose tissue paucity causes diminished endocrine activity. In particular, leptin, with its pleiotropic functions, is a critical endocrine factor for the adequate regulation of glucose homeostasis and energy balance $[4,5]$. An option for LD therapy is the administration of leptin. Indeed, leptin replacement therapy has been shown to improve glucose and lipid homeostasis, as well as fatty liver disease, in several subtypes of LD $[6,7]$.

Due to its molecular mass of only $16 \mathrm{kDa}$, leptin is quickly eliminated from the blood by renal filtration $[8$, 9]. Thus, leptin must be administered at least on a dayto-day basis to achieve therapeutic efficacy. Plasma halflife extension offers a potent strategy to overcome the limitations of most biologics with poor pharmacokinetic properties [10]. Recently, we developed a novel leptin version by fusing a polypeptide comprising 600 Pro, Ala and Ser (PAS) residues to the N-terminus of the murine protein [11]. The PAS moiety adopts a random coil conformation and expands the average diameter of the fusion protein beyond the pore size of the kidney filtration barrier [11-13].

Previous experiments in $\mathrm{C} 57 \mathrm{BL} / 6 \mathrm{~J}$ mice demonstrated a much prolonged plasma half-life, from around $30 \mathrm{~min}$ for the unmodified leptin to $20 \mathrm{~h}$ for PAS-leptin [11]. In a subsequent study with obese leptin-deficient $\mathrm{Lep}^{\text {ob/ob }}$ mice, a dose of only $~ 16$ nmol PAS-leptin (applied via four injections over 20 days) sufficiently induced a weight loss of $>40 \%$ and alleviated glucose intolerance and hepatic steatosis [14]. These superior characteristics of PAS-leptin are further highlighted by comparison with previous studies where a 25 -fold amount of unmodified leptin ( $400 \mathrm{nmol})$, with daily injections, was required to achieve similar effects $[15,16]$.

An established method for inducing LD in mice is feeding a diet supplemented with conjugated linoleic acid (CLA) [17-19]. In CLA fed mice, we compared the efficacy of unmodified and PASylated leptin to alleviate hepatic steatosis and insulin intolerance, and explored the acute effect of PAS-leptin on energy balance.

\section{Methods}

\section{Preparation of recombinant murine leptin and PAS-leptin}

PASylated leptin, comprising a PAS\#1 polypeptide [13] with a length of 600 amino acids, and unmodified leptin were produced in E. coli as described [11].

\section{Animals and experimental design}

All experiments were conducted with permission from the District Government of Upper Bavaria, Germany (License no. 55.2-1-54-2532-183-11) in a specific pathogen-free animal facility. C57BL/6J mice, obtained from an in-house breeding colony (Kleintierforschungszentrum Weihenstephan, TU München, Freising, Germany), were maintained at $22^{\circ} \mathrm{C}$ and relative humidity of $55 \%$ under light/dark $(12 \mathrm{~h} / 12 \mathrm{~h})$ regulated conditions. From the age of 8 weeks onwards, all mice received a purified control diet (CD) containing 5\% wt/wt soy oil (no. S5745-E702; Ssniff, Soest, Germany). Two weeks later, all mice were transferred to single cages and LD was induced by CLA feeding. The CLA diet contained 3.5\% wt/wt soy oil and $1.5 \% \mathrm{wt} / \mathrm{wt}$ tonalin (TG 80; BASF, Lampertheim, Germany) as CLA source. Tonalin contains $80 \%$ vol./vol. CLA with a 50:50 ratio of the two active C18:2 CLA isomers $\mathrm{c} 9, \mathrm{t} 11$ and t10,c12. The CD and CLA diets had the same energy content of $17.4 \mathrm{~kJ} / \mathrm{g}$ and assimilation efficiencies of $90 \%$ (unpublished observations from the authors F. Bolze, A. Bast and M. Klingenspor). After 3 weeks of CLA feeding, mice were body weight matched and injected either with PBS, unmodified leptin or PAS-leptin, while CLA feeding was continued. In Cohort I and II, the injection start was defined as 'day 0 '.

Cohort I Over 11 days, female mice received four s.c. injections of leptin or PAS-leptin (50 pmol/g per injection; aiming at a peak plasma concentration $\left[\mathrm{C}_{\max }\right]$ of 250 $300 \mathrm{nmol} / \mathrm{l}$ ), or a volume of $\sim 80 \mu \mathrm{l}$ PBS every $72 \mathrm{~h}$ (50 pmol corresponds to $0.85 \mu \mathrm{g}$ leptin and $3.35 \mu \mathrm{g}$ PASleptin). At day 5 ( $48 \mathrm{~h}$ after the second injection) insulin levels were assessed. Insulin tolerance was measured at day 8 ( $48 \mathrm{~h}$ after the third injection). At day 11 (48 $\mathrm{h}$ after the fourth injection) mice were dissected.

Cohort II Male mice received one s.c. injection of $50 \mathrm{pmol} / \mathrm{g}$ PAS-leptin or $\sim 80 \mu \mathrm{l}$ PBS. The acute effect of PASylated leptin on energy balance was assessed by parallel measurements of energy intake and energy expenditure for $48 \mathrm{~h}$.

\section{Plasma insulin and leptin quantification and insulin tolerance test}

In Cohort I, insulin levels were analysed at day 5 after $6 \mathrm{~h}$ fasting using a murine insulin ELISA (Mercodia, Uppsala, 
Sweden). At day 8, mice were fasted for $6 \mathrm{~h}$ and received an intraperitoneal injection of $0.45 \mathrm{U} / \mathrm{kg}$ human insulin (Insuman Rapid; Sanofi-Aventis, Frankfurt/Main, Germany). Blood was sampled from a small incision in the tail tip. Glucose levels were quantified with a Freestyle Lite glucometer (Abott, Wiesbaden, Germany).

In a separate cohort of male $\mathrm{C} 57 \mathrm{BL} / 6 \mathrm{~J}$ mice, plasma leptin levels were measured at the end of a 3 week CLA feeding period, using a murine leptin ELISA (Biovendor, Kassel, Germany)

\section{Indirect calorimetry}

In Cohort II, energy expenditure was assessed in an open flow respirometry system (TSE systems, Bad Homburg, Germany). One day prior to the measurement, mice were placed in metabolic cages equivalent to the regular home cage used during the induction phase. Air was drawn from these cages (flow $0.7 \mathrm{l} / \mathrm{min}$ ), dried in a cooling trap and analysed for $\mathrm{O}_{2}$ and $\mathrm{CO}_{2}$ concentrations in 9 min intervals. Respiratory exchange ratio (RER) and heat production were calculated as described previously [20]. Cumulative metabolic rate (MR) represents heat production over $48 \mathrm{~h}$ of the measurement. For data evaluation, cumulative MR was adjusted to the covariate lean mass using the relation $\mathrm{MR}=2.52 \times$ lean mass $+39.1\left(r^{2}=0.271, p<0.05\right)$ [21]. For more precise analysis of substrate oxidation, five arbitrary RER range bins were defined and the duration for which mice maintained their RER within these ranges was computed. The lowest two bins reflected preferential lipid oxidation (0.70-0.75 and 0.76-0.81), whereas the higher ones indicated more carbohydrate oxidation $(0.88-0.93$ and 0.94 1.00). Accordingly, balanced fuel selection was indicated by the intermediate range (0.82-0.87). Carbohydrate and fatty acid oxidation were determined from RER and cumulative MR [22].

\section{Energy intake, body mass and body composition}

Food intake was quantified by weighing of the food racks, and converted to $\mathrm{kJ}$ for assessment of energy intake. In the induction phase, energy intake for both cohorts was assessed weekly. During the treatment phase, energy intake for Cohort I was determined every 3 days and, for Cohort II, $48 \mathrm{~h}$ after the injection. Body mass, total body fat and total body lean mass (LF50H TD-NMR Analyzer; Bruker Biospin, Rheinstetten, Germany) were repeatedly measured (days for Cohort I: $-21,-14,-7,0,7,11$ and for Cohort II: $-21,-14,-7,-1)$.

\section{Liver phenotyping}

In Cohort I, hepatosteatosis was evaluated via inspection of haematoxylin/eosin (HE) stained sections, by three 'blinded' collaborators utilising a light microscopy histoscore ranging from 0 (no steatosis) to 4 (severe steatosis). Hepatic triacylglycerol content was assayed with an enzymatic test based on the GPO/PAP method (Triglycerides Liquicolor; Human, Wiesbaden, Germany; see electronic supplementary material [ESM]). Plasma concentrations of the liver enzymes alanine aminotransferase (ALT) and aspartate aminotransferase (AST) were measured using commercially available colourimetric kits (ALTPM and ASTPM; Roche Diagnostics, Indianapolis, IN, USA).

\section{Data handling and statistics}

The design included six to seven mice per group. In Cohort I some variables could not be assessed in all mice due to experimental constraints, which may represent a potential limitation of this study. Hypoglycaemia after $6 \mathrm{~h}$ fasting prevented insulin tolerance tests in two mice, and blood sample volume was insufficient for insulin ELISA in two cases. After the CLA induction phase, one growth-retarded mouse and one AST value were excluded from statistical analysis based on Grubb's outlier test. Data are expressed as means \pm SD. Statistical significance of normally distributed data was tested by either unpaired $t$ tests or one-way ANOVA with subsequent multiple comparisons using Sigmaplot 12.5 (Systat Software, Erkrath, Germany). Non-normally distributed data were $\log _{10^{-}}$ transformed.

\section{Results}

\section{CLA feeding induces LD in C57BL/6J mice}

Feeding a CLA-diet for 3 weeks induced LD in both sexes, as demonstrated by a loss in total body fat; compared with the CDfed groups, body fat mass was reduced by $54 \%$ in Cohort I and $38 \%$ in Cohort II of CLA-fed (LD) mice (ESM Table 1 and ESM Fig. 1). Also, CLA feeding resulted in hypoleptinaemia (CD: $122 \pm 70 \mathrm{pmol} / 1$ vs CLA: $44 \pm 24 \mathrm{pmol} / 1, p<0.001)$, as seen in a separate cohort of male mice with comparable body fat reduction (data not shown). Body mass was similar in CD- and CLAfed mice since fat loss was accompanied by a gain in lean mass in the CLA-groups (ESM Table 1 and ESM Fig. 1). Notably, energy density of fat tissue is nearly twice that of lean tissue. Hence, this shift in body composition should be associated with a loss in total body energy content, indicating that CLA induced a negative energy balance. Therefore, CLA most likely accelerated energy expenditure, since energy intake was similar between CD- and CLA-fed mice (ESM Table 1 and ESM Fig. 2).

\section{Repeated PAS-leptin injections normalise insulin intolerance and hepatic steatosis}

To compare the efficacy of conventional leptin with PAS-leptin, female LD mice (Cohort I) received four periodic s.c. 
injections every $72 \mathrm{~h}$, distributed over 11 days. The total protein dose of $\sim 4 \mathrm{nmol} /$ mouse matches the amount of conventional leptin infused by osmotic pumps over 12 days in previous studies with CLA mice $[18,19]$. Relative to the CD/PBS group, CLA feeding with PBS injections almost doubled fasting plasma insulin levels (Fig. 1a). In contrast, treatment of CLA fed mice with PAS-leptin normalised plasma insulin, to concentrations similar to those measured in $\mathrm{CD} / \mathrm{PBS}$ mice (Fig. 1a). CLA/PBS mice exhibited altered glucose trajectories after insulin administration, resulting in a $\sim 60 \%$ larger area under the curve compared with CD/PBS animals (Fig. 1b, c). Administration of PAS-leptin corrected impaired insulin sensitivity in LD mice to similar levels to those observed in the CD/PBS group whereas unmodified leptin was ineffective in normalising both plasma insulin levels and insulin tolerance (Fig. 1a, c).

Moreover, relative to CD/PBS, CLA feeding with PBS injections increased liver weight ( twofold, Fig. 2a), hepatic triacylglycerol content ( fourfold, Fig. 2b), liver vacuolisation (Fig. 2c, d) and plasma liver enzyme activities in LD mice (Table 1). Compared with CLA/PBS mice, liver weight and triacylglycerol content in the CLA/PAS-leptin group were reduced by approximately $25 \%$ and $60 \%$, respectively (Fig. 2a, b). The vacuolisation status in the CLA/PAS-leptin group also approached the degree observed for the CD/PBS group (Fig. 2c, d). For liver enzymes, however, only a non-significant trend towards normalisation was observed (Table 1). As seen with insulin levels and insulin sensitivity, unmodified leptin also did not mediate improvements on hepatosteatosis (Fig. 2).

Compared with CLA/PBS and CLA/leptin groups, PASleptin led to a loss of around $0.5 \mathrm{~g}$ in total body fat in LD mice (Table 1) which was contributed by reductions in liver weight by $\sim 0.5 \mathrm{~g}$ (Fig. 2a) and in gonadal adipose tissue (Table 1). Relative to the other CLA-fed groups, the PAS-leptin group had a significantly lower average daily energy intake during the treatment period (CLA/PBS mice: $44.8 \pm 3.6 \mathrm{~kJ} / \mathrm{day}$, CLA/ Leptin mice: $48.3 \pm 2.5 \mathrm{~kJ} /$ day, CLA/PAS-leptin mice: 41.8 $\pm 2.7 \mathrm{~kJ} /$ day; $p<0.05$ ).

\section{A single PAS-leptin injection has no effect on MR but promotes fat utilisation}

We explored the effect of a single s.c. injection of either PBS or PAS-leptin on energy balance in male LD mice (Cohort II). All treatment groups showed an equal diurnal rhythm of heat production, with highest values in the dark and lowest during light phase (Fig. 3a). Compared with CD/PBS mice, both CLA groups had a $\sim 10 \%$ increased cumulative MR (Fig. 3b). This elevated energy expenditure in LD mice was attenuated after adjusting for lean mass which was higher in both groups of CLA fed LD mice (Fig. 3cd). In CLA/PASleptin mice, however, higher lean mass did not completely explain increased energy expenditure as adjusted cumulative MR remained elevated by $\sim 5 \%$ compared to $\mathrm{CD} / \mathrm{PBS}$ mice (Fig. 3d). Regarding the other side of the energy balance equation, PAS-leptin induced a statistically significant reduction of energy intake by approximately $14 \%$ in LD mice compared with PBS (CLA/PBS: $115.0 \pm 9.3 \mathrm{~kJ} / 48 \mathrm{~h}$ vs CLA/PAS-leptin: $98.6 \pm 9.3 \mathrm{~kJ} / 48 \mathrm{~h} ; p<0.05)$.

Diurnal RER amplitudes, as well as mean dark and light RERs differed between the CD/PBS and CLA/PBS groups (Fig. 4a). Consistently, relative to CD/PBS mice, the CLA/ PBS group spent less time within 'extreme' RER ranges, which indicate 'pure fat' or 'pure carbohydrate' oxidation (Fig. 4b). However, the overall $48 \mathrm{~h}$ mean RER was not altered between CD/PBS and CLA/PBS mice and, consistently, overall fuel utilisation did not differ between the two groups (Fig. 4a, c). Treatment with PAS-leptin altered the RER a

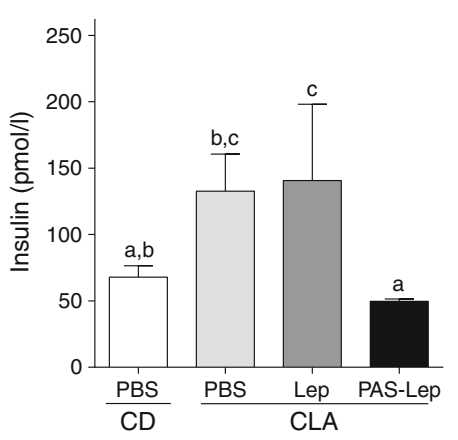

b

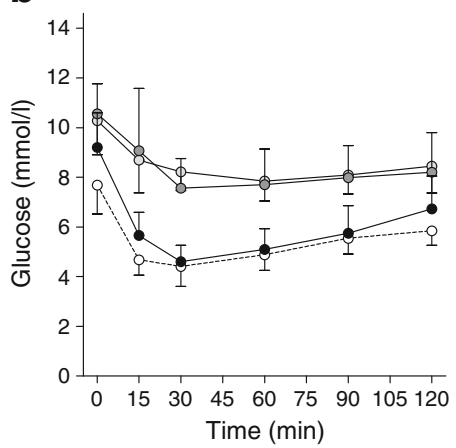

C

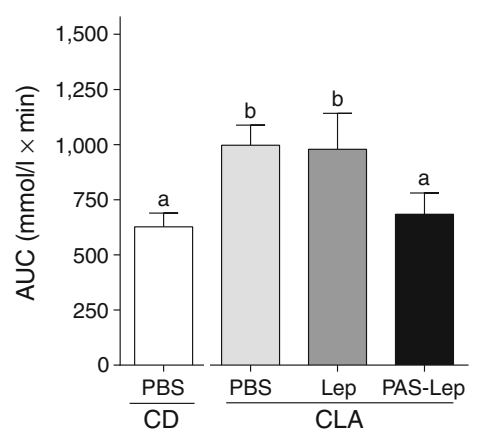

Fig. 1 Cohort I: effect of repeated PBS, leptin and PAS-leptin injections on insulin levels and insulin tolerance. Insulin levels were assessed after the second injection and insulin tolerance test (ITT) was performed after the third injection. After 3 weeks of LD induction by LA, mice received four s.c. injections of either leptin, PAS-leptin or PBS. (a) Plasma insulin levels $(n=3-6)$. (b) Blood glucose trajectories during ITT $(n=5-6)$. (c)
Corresponding ITT area under the curve $(n=5-6)$. Data are expressed as means with SD. Non-matching lowercase letters indicate a statistically significant difference identified by one-way ANOVA and Holm-Sidak multiple comparison test $(p<0.05)$. White, CD/PBS; light grey, CLA/ PBS; intermediate grey, CLA/Lep; black, CLA/PAS-Lep 
a

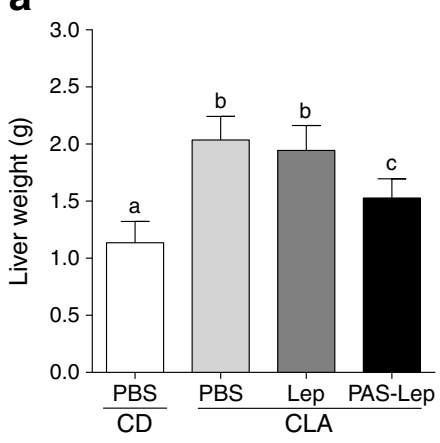

b

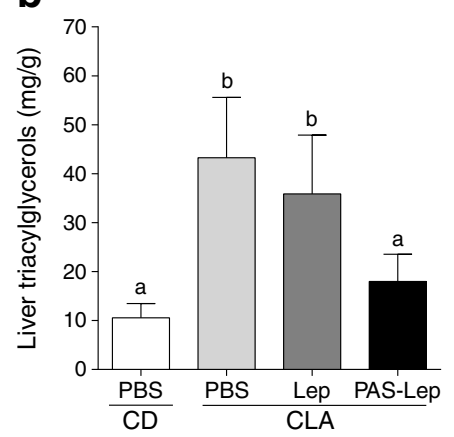

C

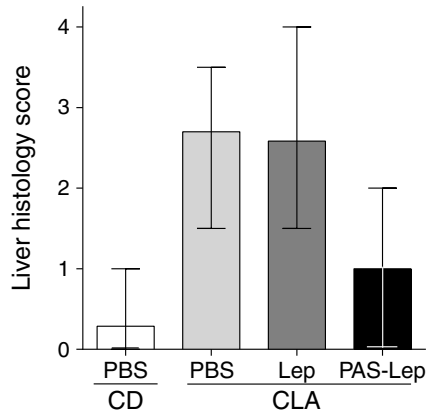

d

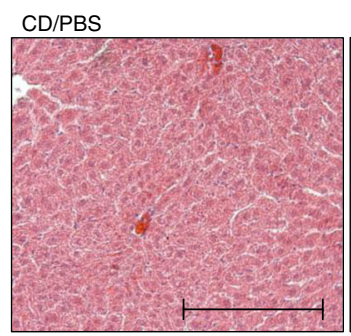

CLA/PBS

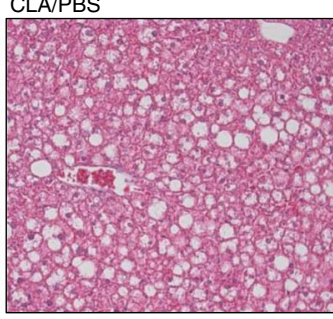

CLA/Lep

CLA/PAS-Lep
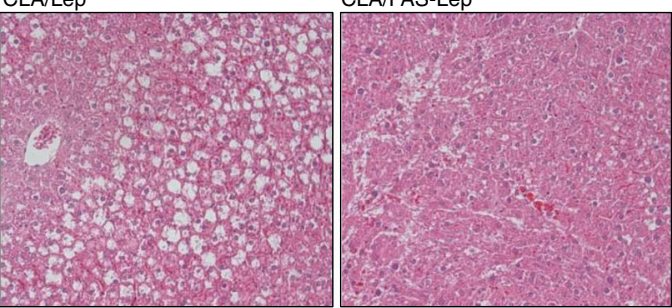

Fig. 2 Cohort I: effect of repeated PBS, leptin and PAS-leptin injection on hepatosteatosis. After LD induction by CLA, mice were treated over 11 days with four repeated s.c. injections of leptin, PAS-leptin or PBS. Endpoint liver variables were assessed after the fourth injection. (a) Liver weight $(n=5-7)$. (b) Liver triacylglycerols $(n=5-7)$. (c) Liver histology score from the evaluation of HE stained sections $(n=5-7)$. Data in (a) and (b) are expressed as means with SD. In (c) data are expressed as means

trajectory and lowered the mean RER over $48 \mathrm{~h}$ in CLA fed mice (Fig. 4a). CLA/PAS-leptin mice spent more time within lower RER ranges relative to CLA/PBS mice (Fig. 4b). Accordingly, this alteration resulted in overall higher fat and lower carbohydrate utilisation in CLA/PAS-leptin mice, relative to the two other groups (Fig. 4c). \pm range which indicates minimal and maximal values. Non-matching lowercase letters indicate a statistically significant difference identified by one-way ANOVA and Holm-Sidak multiple comparison test $(p<0.05)$. White, CD/PBS; light grey, CLA/PBS; intermediate grey, CLA/Lep; black, CLA/PAS-Lep. (d) Representative HE stained liver sections (200-fold magnification, scale bar $200 \mu \mathrm{m}$ )

\section{Discussion}

PASylation has allowed the design of biopharmaceuticals with tailored pharmacological properties to improve animal studies, as well as therapies and diagnosis of various diseases [13, $23,24]$. Here, we explored the efficacy of a new long-acting
Table 1 Leptin treatment effects on body mass, body composition, organ weights and liver enzymes in LD Cohort I

\begin{tabular}{lcccc}
\hline Variable & CD & \multicolumn{2}{l}{ CLA } & \\
\cline { 3 - 4 } & PBS $(n=7)$ & PBS $(n=5)$ & Leptin $(n=6)$ & PAS-leptin $(n=6)$ \\
\hline Body mass (g) & $20.3 \pm 1.4$ & $20.2 \pm 0.7$ & $20.1 \pm 0.4$ & $18.9 \pm 1.1$ \\
Lean mass (g) & $14.9 \pm 1.1$ & $15.8 \pm 0.3$ & $16.1 \pm 0.6$ & $15.5 \pm 1.1$ \\
Fat mass (g) & $2.82 \pm 0.36^{\mathrm{a}}$ & $1.46 \pm 0.26^{\mathrm{b}}$ & $1.55 \pm 0.18^{\mathrm{b}}$ & $0.97 \pm 0.30^{\mathrm{c}}$ \\
Inguinal WAT (mg) & $214 \pm 35^{\mathrm{a}}$ & $44 \pm 6^{\mathrm{b}}$ & $48 \pm 9^{\mathrm{b}}$ & $45 \pm 6^{\mathrm{b}}$ \\
Gonadal WAT (mg) & $215 \pm 58^{\mathrm{a}}$ & $114 \pm 35^{\mathrm{b}}$ & $119 \pm 25^{\mathrm{b}}$ & $46 \pm 16^{\mathrm{c}}$ \\
Interscapular BAT (mg) & $58 \pm 8^{\mathrm{a}}$ & $14 \pm 1^{\mathrm{b}}$ & $15 \pm 2^{\mathrm{b}}$ & $13 \pm 3^{\mathrm{b}}$ \\
Quadriceps (mg) & $98 \pm 0.018$ & $102 \pm 27$ & $85 \pm 12$ & $90 \pm 11$ \\
AST (U/l) & $125 \pm 45$ & $354 \pm 231$ & $281 \pm 251$ & $193 \pm 70$ \\
ALT (U/l) & $40 \pm 24^{\mathrm{a}}$ & $217 \pm 132^{\mathrm{b}}$ & $198 \pm 137^{\mathrm{b}}$ & $118 \pm 57^{\mathrm{b}}$ \\
\hline
\end{tabular}

Data are expressed as means $\pm \mathrm{SD}$

Endpoint variables were assessed after 11 days of treatment. During treatment, each mouse received four s.c. injections every $72 \mathrm{~h}$ of either leptin, PAS-leptin or PBS

Lean and fat mass do not equate to body mass because the NMR technique does not detect bones, fur, teeth and blood

Statistical differences between values were assessed by one way ANOVA and Holm-Sidak multiple comparison test. Non-matching lowercase letters indicate a statistically significant difference among groups $(p<0.05)$ 


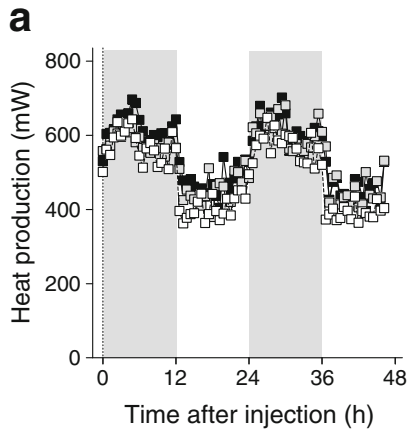

C

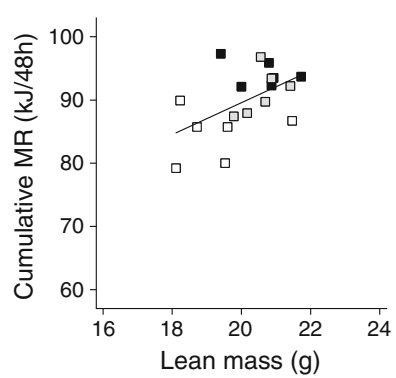

Fig. 3 Cohort II: effect of a single PBS or PAS-leptin injection on energy expenditure. After 3 weeks of LD induction by CLA, mice were placed in an indirect calorimetry system. At time point ' 0 ', mice received a single PBS or PAS-leptin injection. (a) Heat production; shaded regions indicate dark cycle $(n=6)$. (b) Cumulative MR $(n=6)$. (c) $48 \mathrm{~h}$ cumulative MR, plotted against lean mass (assessed on day -1) (d) Cumulative MR adjusted to lean mass $(n=6)$. Data are expressed as means with SD. Nonmatching lower case letters indicate a statistically significant difference identified by one-way ANOVA and Holm-Sidak multiple comparison test $(p<0.05)$. White, CD/PBS; light grey, CLA/PBS; black, CLA/PASLep

leptin version prepared via PASylation in a CLA-induced lipodystrophic (LD) mouse model. The supremacy of PASleptin was demonstrated by efficient normalisation of insulin sensitivity, plasma insulin levels and fatty liver disease. These metabolic improvements are in line with previous reports in which conventional leptin - infused via osmotic pumps successfully ameliorated LD symptoms in CLA fed mice $[18,19]$. The failure of unmodified leptin, with its poor pharmacokinetic characteristics, to induce metabolic benefits was not surprising at the low dose applied and the rather long injection intervals. Conversely, the treatment regimen was perfectly suited for PAS-leptin and suggests benefits in clinical settings. It is worth mentioning that not only LD patients should profit from PAS-leptin; several preclinical studies in mice have suggested that individuals suffering from type 1 and type 2 diabetes or neurodegenerative diseases may also benefit from optimised leptin analogues [25-27].

No previous study has focused on the acute effect of leptin on energy balance in LD mice. PAS-leptin shifted fuel selection towards lipid oxidation. This could have been mediated either by direct stimulation of lipid metabolism or by an inhibitory effect on energy intake [28-30]. Indeed, the latter
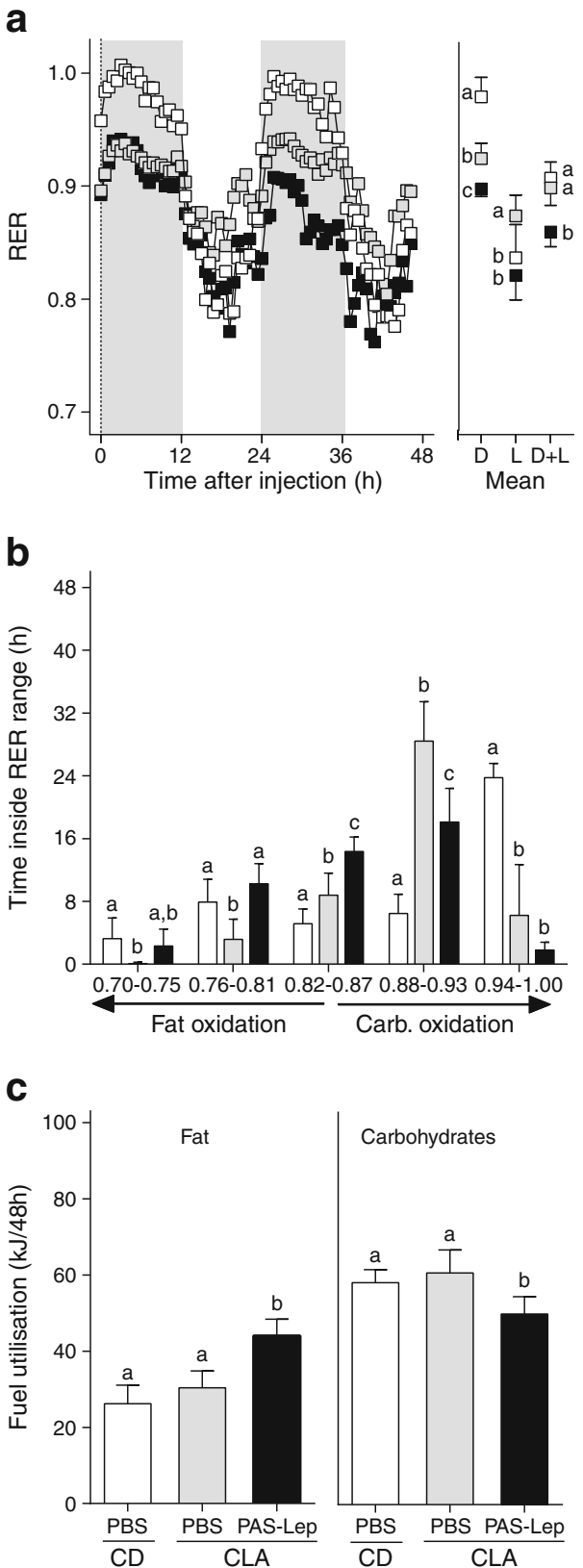

Fig. 4 Cohort II: effect of a single PBS or PAS-leptin injection on fuel selection. After LD induction by CLA, mice were placed for $48 \mathrm{~h}$ in an indirect calorimetry system. At time point ' 0 ' mice received a single injection of PBS or PAS-leptin. (a) RER trajectories and mean values for dark cycle (D), light cycle (L) and both phases $(\mathrm{D}+\mathrm{L})(n=6)$. (b) Hours spent within the five arbitrary RER ranges $(n=6)$. (c) $48 \mathrm{~h}$ fuel utilisation $(n=6)$. Data are expressed as means with SD. Statistical analysis was conducted by one-way ANOVA and Holm-Sidak multiple comparison test (in $\mathbf{b}$ an ANOVA was used within each RER range). Nonmatching lowercase letters indicate a statistically significant difference $(p<0.05)$. White, CD/PBS; light grey, CLA/PBS; black, CLA/PASLep

phenomenon was confirmed in both cohorts used in this study. Accordingly, food intake did not provide sufficient calories, thus promoting the oxidation of endogenous fat stores. As a consequence, body fat was decreased in Cohort I. The 
majority of this body fat loss can be attributed to the reduction of liver fat, thus linking the increased fat utilisation observed during indirect calorimetry to the correction of a pathological hallmark of LD. Apart from decreased energy intake, a direct impact of PAS-leptin on lipid utilisation, which would further accelerate the elimination of ectopic fat, cannot be excluded. In fact, in the genetic aP2-SREBP-1c LD mouse model a 30\% food restriction was not as effective as leptin infusion, which supports both a direct and indirect role of leptin on the amelioration of hepatosteatosis [31].

Low plasma leptin levels are known to mediate metabolic suppression $[4,32]$. Counterintuitively, hypoleptinaemia (induced by CLA feeding) was associated with increased cumulative MR. As CLA did not affect energy intake, elevated cumulative MR is obviously a valid explanation for the reduction in total body energy content indicated by lower body fat of LD mice. Likewise, others have reported an increase in energy expenditure during CLA feeding in LD mice [33-36]. However, some of these findings must be critically re-evaluated due to pitfalls in the use of mass-specific ratios to normalise for differences in body weight and body composition $[34,35]$. Mass-specific ratios can lead to over- or underestimations of MR [37]. By state-of-the-art data analysis, we demonstrate that increased cumulative MR in LD mice was due to the higher lean mass induced by CLA feeding, since adjustment for variation in lean mass either removed or attenuated the significant differences. Our analysis of cumulative MR revealed no explicit tachymetabolic effect of PAS-leptin. In CLA/PAS-leptin mice, lean mass-adjusted cumulative MR remained significantly elevated compared with $\mathrm{CD} / \mathrm{PBS}$ mice but was not different from the CLA/PBS group. At the given difference of less than $5 \%$ between CLA/PBS and CD/PASleptin mice, a power calculation indicates that the sample size must be increased to $>20$ mice per group in order to verify whether PAS-leptin may stimulate energy expenditure in LD mice, hence leaving this question for future study.

In conclusion, a remarkably low dose of $\sim 4 \mathrm{nmol}$ longacting PASylated leptin alleviated insulin intolerance and hepatosteatosis in LD mice. PAS-leptin altered fuel selection at least partially by a reduction of energy intake but may also operate through direct stimulation of sympathetic nervous system activity [38] or peripheral effects on lipolysis [28-30]. In concert with our previous reports [11, 14], these findings underline the superiority of PAS-leptin in preclinical research and its potential for therapeutic application.

Funding MH was supported by a European Research Council (ERC) grant (LiverCancerMech).

Duality of interest AS and MS are shareholders of XL-protein GmbH.

Contribution statement FB designed the experiments, acquired and analysed data and drafted the manuscript. AB, SM, VM, DY, NR, MS and $\mathrm{AZ}$ acquired data and drafted the manuscript. MH contributed to the study design and drafted the manuscript. AS and MK drafted the manuscript, critically revised the text and contributed to the study design and data analysis. All authors approved the final version of the text. FB is responsible for the integrity of the work as a whole.

\section{References}

1. Garg A (2011) Lipodystrophies: genetic and acquired body fat disorders. J Clin Endocrinol Metab 96:3313-3325

2. Trayhurn P, Beattie JH (2001) Physiological role of adipose tissue: white adipose tissue as an endocrine and secretory organ. Proc Nutr Soc 60:329-339

3. Ahima RS (2006) Adipose tissue as an endocrine organ. Obesity (Silver Spring) 14(Suppl 5):242S-249S

4. Halaas JL, Boozer C, Blair-West J, Fidahusein N, Denton DA, Friedman JM (1997) Physiological response to long-term peripheral and central leptin infusion in lean and obese mice. Proc Natl Acad Sci U S A 94:8878-8883

5. Koch C, Augustine RA, Steger J et al (2010) Leptin rapidly improves glucose homeostasis in obese mice by increasing hypothalamic insulin sensitivity. J Neurosci 30:16180-16187

6. Oral EA, Simha V, Ruiz E et al (2002) Leptin-replacement therapy for lipodystrophy. N Engl J Med 346:570-578

7. Chong AY, Lupsa BC, Cochran EK, Gorden P (2010) Efficacy of leptin therapy in the different forms of human lipodystrophy. Diabetologia 53:27-35

8. Cumin F, Baum HP, Levens N (1996) Leptin is cleared from the circulation primarily by the kidney. Int J Obes Relat Metab Disord 20:1120-1126

9. Zhang F, Basinski MB, Beals JM et al (1997) Crystal structure of the obese protein leptin-E100. Nature 387:206-209

10. Kontermann RE (2011) Strategies for extended serum half-life of protein therapeutics. Curr Opin Biotechnol 22:868-876

11. Morath V, Bolze F, Schlapschy M et al (2015) PASylation of murine leptin leads to extended plasma half-life and enhanced in vivo efficacy. Mol Pharm 12:1431-1442

12. Haraldsson B, Sorensson J (2004) Why do we not all have proteinuria? An update of our current understanding of the glomerular barrier. News Physiol Sci 19:7-10

13. Schlapschy M, Binder U, Börger C et al (2013) PASylation: a biological alternative to PEGylation for extending the plasma half-life of pharmaceutically active proteins. Protein Eng Des Sel 26:489-501

14. Bolze F, Morath V, Bast A et al (2016) Long-acting PASylated leptin ameliorates obesity by promoting satiety and preventing hypometabolism in leptin-deficient Lep(ob/ob) mice. Endocrinology 157:233-244

15. Pelleymounter MA, Cullen MJ, Baker MB et al (1995) Effects of the obese gene product on body weight regulation in ob/ob mice. Science 269:540-543

16. Halaas JL, Gajiwala KS, Maffei M et al (1995) Weight-reducing effects of the plasma protein encoded by the obese gene. Science 269:543-546

17. Park Y, Albright KJ, Liu W, Storkson JM, Cook ME, Pariza MW (1997) Effect of conjugated linoleic acid on body composition in mice. Lipids 32:853-858

18. Tsuboyama-Kasaoka N, Takahashi M, Tanemura K et al (2000) Conjugated linoleic acid supplementation reduces adipose tissue by apoptosis and develops lipodystrophy in mice. Diabetes 49 : 1534-1542 
19. Nagao K, Inoue N, Ujino Y et al (2008) Effect of leptin infusion on insulin sensitivity and lipid metabolism in diet-induced lipodystrophy model mice. Lipids Health Dis 7:8

20. Heldmaier G, Ruf T (1992) Body temperature and metabolic rate during natural hypothermia in endotherms. J Comp Physiol B 162: 696-706

21. Tschöp MH, Speakman JR, Arch JR et al (2012) A guide to analysis of mouse energy metabolism. Nat Methods 9:57-63

22. Elia M, Livesey G (1992) Energy expenditure and fuel selection in biological systems: the theory and practice of calculations based on indirect calorimetry and tracer methods. World Rev Nutr Diet 70: 68-131

23. Mendler CT, Friedrich L, Laitinen I et al (2015) High contrast tumor imaging with radio-labeled antibody Fab fragments tailored for optimized pharmacokinetics via PASylation. MAbs 7:96-109

24. Harari D, Kuhn N, Abramovich R et al (2014) Enhanced in vivo efficacy of a type I interferon superagonist with extended plasma half-life in a mouse model of multiple sclerosis. J Biol Chem 289: 29014-29029

25. Naito M, Fujikura J, Ebihara K et al (2011) Therapeutic impact of leptin on diabetes, diabetic complications, and longevity in insulindeficient diabetic mice. Diabetes 60:2265-2273

26. Toyoshima Y, Gavrilova O, Yakar S et al (2005) Leptin improves insulin resistance and hyperglycemia in a mouse model of type 2 diabetes. Endocrinology 146:4024-4035

27. Perez-Gonzalez R, Alvira-Botero MX, Robayo O et al (2014) Leptin gene therapy attenuates neuronal damages evoked by amyloid- $\beta$ and rescues memory deficits in APP/PS1 mice. Gene Ther 21:298-308

28. Wang MY, Lee Y, Unger RH (1999) Novel form of lipolysis induced by leptin. J Biol Chem 274:17541-17544
29. Minokoshi Y, Kim YB, Peroni OD et al (2002) Leptin stimulates fatty-acid oxidation by activating AMP-activated protein kinase. Nature 415:339-343

30. Huang W, Dedousis N, Bandi A, Lopaschuk GD, O'Doherty RM (2006) Liver triglyceride secretion and lipid oxidative metabolism are rapidly altered by leptin in vivo. Endocrinology 147:1480-1487

31. Shimomura I, Hammer RE, Ikemoto S, Brown MS, Goldstein JL (1999) Leptin reverses insulin resistance and diabetes mellitus in mice with congenital lipodystrophy. Nature 401:73-76

32. Döring H, Schwarzer K, Nuesslein-Hildesheim B, Schmidt I (1998) Leptin selectively increases energy expenditure of food-restricted lean mice. Int J Obes Relat Metab Disord 22:83-88

33. West DB, Delany JP, Camet PM, Blohm F, Truett AA, Scimeca J (1998) Effects of conjugated linoleic acid on body fat and energy metabolism in the mouse. Am J Physiol 275:R667-R672

34. West DB, Blohm FY, Truett AA, DeLany JP (2000) Conjugated linoleic acid persistently increases total energy expenditure in AKR/ $\mathrm{J}$ mice without increasing uncoupling protein gene expression. J Nutr 130:2471-2477

35. Ohnuki K, Haramizu S, Ishihara K, Fushiki T (2001) Increased energy metabolism and suppressed body fat accumulation in mice by a low concentration of conjugated linoleic acid. Biosci Biotechnol Biochem 65:2200-2204

36. Terpstra AH, Beynen AC, Everts H, Kocsis S, Katan MB, Zock PL (2002) The decrease in body fat in mice fed conjugated linoleic acid is due to increases in energy expenditure and energy loss in the excreta. J Nutr 132:940-945

37. Rozman J, Klingenspor M, Hrabe de Angelis M (2014) A review of standardized metabolic phenotyping of animal models. Mamm Genome 25:497-507

38. Zeng W, Pirzgalska RM, Pereira MM et al (2015) Sympathetic neuroadipose connections mediate leptin-driven lipolysis. Cell 163:84-94 